\title{
PFC Fuzzy Decision-Making Control and Its Application to Car-Like Mobile Vehicle
}

\author{
You-gen Chen ${ }^{1}$, Seiji Yasunobu ${ }^{2}$, Wei-hua Gui ${ }^{1}$, \\ Ren-yong $\mathrm{Wei}^{1}$ and Zhi-yong $\mathrm{Li}^{1}$ \\ ${ }^{1}$ Central South University, China \\ ${ }^{2}$ University of Tsukuba, Japan
}

\section{Introduction}

Human have a remarkable capability to perform a wide variety of physical and mental tasks without any measurements and any computations. Underlying this capability is brain's crucial ability to manipulate perceptions and remarkable capability to operate on and reason with, perception-based information which are intrinsically vague, imprecise and fuzzy. Human's action decision (Fig. 1) is based on multi-targets and can respond flexibly under different situations just based on information which are intrinsically vague, imprecise and fuzzy. The best alternative target is selected in real time based on experiences by predicting and evaluating the object's states with taking dynamic environment information into account.

From the process of human decision, it is clear that the multi-targets-based methodology results in their dynamic soft decision and flexibility rather than single-target-based conventional method. The image of multi-targets and its utilization is denoted in Fig. 2, when disturbances make the best target $r_{3}$ in multi-target set with membership 1.0 become unavailable, system selects the sub-optimal target $r_{2}$ as control target automatically to respond to situation change in environment.

For a real control system, the fact is that there usually exist plural targets (all possible and reachable sub-targets) with their membership values (reflecting the target how good or bad) for the object to select at all time. Thus, it is possible to provide multi-targets for controlled object rather than single target like the conventional method. This provides a quite important premise for mobile body to act like human and adapt to changing situation flexibly.

Fuzzy logic which firstly introduced by Lotfi Zadeh provides a theoretic foundation for combining multi-targets with fuzzy set to form a target set with membership values reflecting satisfaction degree (how good or bad) of its elements. The target set is defined as "soft target" in this chapter.

Decision making problems are described as applying approximate reasoning and incomplete or uncertain information to find a fuzzy set of decision alternatives and choose the best one from possible alternatives (Bellman \& Zadeh, 1970). Fuzzy control presents a formal methodology for representing, manipulating, and implementing a human's heuristic knowledge about how to control a system, which make it possible to incorporate the knowledge into a fuzzy controller that emulates the decision-making process of the human. Especially, 


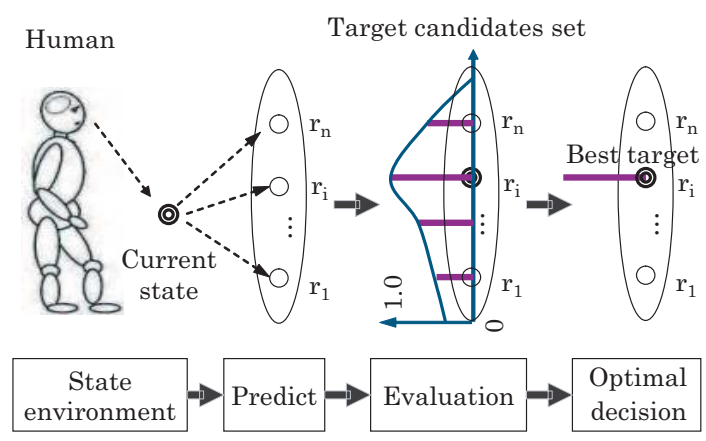

Fig. 1. Decision process of human with fuzzy multi-targets

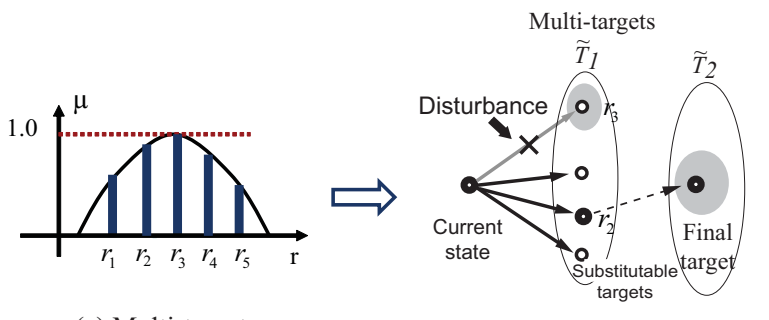

(a) Multi-targets

(b) Multi-targets utilization image

Fig. 2. Image of multi-targets and its utilization

predictive fuzzy control (PFC) method proposed by Prof. Yasunobu provides a quite potential means to realize human's prediction, evaluation and optimal decision-making process to select the best target element from a soft target set by using fuzzy multiple criteria decision making.

In many control systems, the situations always change with the constraints or disturbances. Responding flexibly to the dynamic situation changes in the external world like human is very necessary but difficult for an autonomous control system (Barraquand et al., 1992; Jing, 2005). The motion control of mobile vehicle with dynamic constraints (static and dynamic obstacles) is still a difficult and challenging problem because the surrounding situations are not qualified in static, knowledge is only partial and the execution is often associated with uncertainty.

Therefore, responding flexibly to the situation changes like human for mobile vehicle is considered in this chapter. We proposed a soft-target-based fuzzy decision-making control method to realize flexible autonomous operation like human for motion control of mobile vehicle in a narrow space with change situations to verify the validity of the proposed method. The soft target including many target elements with different satisfaction grade is a multitarget set defined with fuzzy logic. Fuzzy decision-making is realized by predictive fuzzy control to emulate human's prediction, evaluation and optimal decision-making process. The experiment system is mainly composed of a control PC, a CCD camera, and a reconstructed vehicle from a RC car. Simulation and experiment results demonstrate the validity and flexibility of the proposed soft-target-based fuzzy decision-making control method. Collision-free 


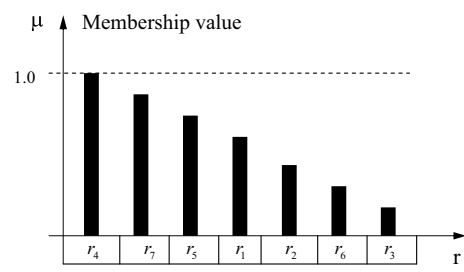

(a) One of soft targets

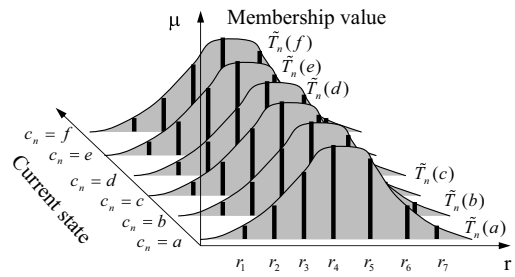

(b) Target setting knowledge

Fig. 3. Definition of soft target

and low cost motion control is achieved, and realized flexible adaptation to changing situations like human.

\section{Soft-Target-Based PFC Fuzzy Decision Making Control}

\subsection{Definition of Soft Target}

Fuzzy logic provides a mathematical strength to capture the uncertainties associated with human cognitive processes, such as thinking and reasoning. It gives us an inference morphology that enables approximate human reasoning capabilities to be applied to knowledge-based systems. Fuzzy set is a means of representing and manipulating data that was not precise, but rather fuzzy.

Fuzzy logic guarantees a theoretic foundation for combining multi-targets with fuzzy set to form a target set with membership values reflecting satisfaction degree (how good or bad) of its elements (Ordonez \& Zumberge, 1997). The target set is defined as "soft target" in this chapter (Chen \& Yasunobu, 2007b).

Soft target is defined as a target set and is converted into target setting knowledge by soft computing. It is constructed from all available target candidates by combining with fuzzy logic based on the final target and constraint information, and can be expressed as a control target set defined by fuzzy set, which includes many alternative candidates. Each candidate has its membership value defined as satisfaction grade in $[0,1]$.

It is denoted as Fig. 3 (a), and can be expressed by the membership function of enumeration type in a discrete space (Chen \& Yasunobu, 2006).

The total set of the target is assumed as $R$. Soft target $\widetilde{T_{n}}$ assumed to be a control target set can be defined by the following expression in state $c_{n}$ of the object.

$$
\widetilde{T_{n}}=\int_{R} \mu_{\widetilde{T_{n}}}\left(r_{i}\right) / r_{i} \quad r_{i} \in R
$$

Here, $\mu_{\widetilde{T_{n}}}\left(r_{i}\right)$ is the membership value of alternative $r_{i}$ in $\widetilde{T_{n}}$ corresponding with state $c_{n}$, and the integral denotes the union of the fuzzy singletons $\mu_{\widetilde{T_{n}}}\left(r_{i}\right) / r_{i}, r_{i} \in R$.

As shown in Fig. 3 (b), target setting knowledge can be expressed as set clusters which correspond with different states. According to different "current" state $c_{n}(\mathrm{a} \sim \mathrm{f})$, the soft target candidates set is $\widetilde{T_{n}}(\mathrm{a} \sim \mathrm{f})$ respectively. Once the current soft target is set, it is possible for the system to select the best alternative candidate instruction corresponding with one of the substitutable target element $r_{i}$ by decision-making. This is repeated until final target or mission achievement. 


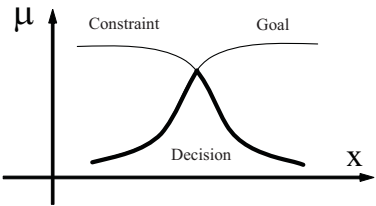

(a) Decision from constraint and goal

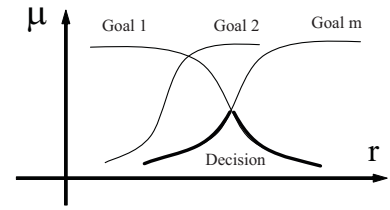

(b) Generalized decision making image

Fig. 4. Decision-making from fuzzy goal and constraint

By using soft target, it is possible to construct an intelligent controller based on multi-targets for a system with dynamic constraints to achieve a flexible operation.

\subsection{PFC Fuzzy Decision-Making}

Decision making problems are described as applying approximate reasoning and incomplete or uncertain information to find a fuzzy set of decision alternatives and choose the best one from possible alternatives. Multi-criteria decision making (MCDM) involves determining the optimal alternative among multiple, conflicting, and interactive criteria, such control goals and external constraints (Kim \& Cho, 2006).

\subsubsection{Fuzzy Decision-Making}

Fuzzy goals and fuzzy constraints can be defined precisely as fuzzy sets in the space of alternatives. A fuzzy decision, then, may be viewed as as intersection of the given goals and constraints. A maximizing decision is defined as a point in the space of alternatives as which the membership function of a fuzzy decision attains its maximum value.

As shown in Fig. 4 (a), assume that we are given a fuzzy goal $G$ and a fuzzy constraint $C$ in a space of alternatives $X$. $G$ and $C$ combine to form a decision, $D$, which is a fuzzy set resulting from intersection of $G$ and $C$.

$$
D=G \cap C
$$

The membership function of the intersection is given by

$$
\mu_{D}=\mu_{G} \wedge \mu_{C}
$$

Then, a maximizing decision can be made from the acquired fuzzy decision set by choosing the one with maximal $\mu$. Fuzzy multi-criteria decision making (FMCDM) which combines MCDM with fuzzy logic provides a promising theoretical framework for alternatives or candidates selection decision.

\subsubsection{Predictive Fuzzy Control}

Predictive fuzzy control is an intelligent control method based on human control strategy. As shown in Fig. 5, firstly, a series of control instruction candidates are prepared based on expert's experience. Next, the future state of controlled object is predicted by using all the instruction candidates in parallel. Then the future state is evaluated by fuzzy inference using expert's knowledge described by fuzzy rules. Lastly, the operation instruction candidate with the highest evaluation value is selected as the current control instruction. Consequently, an 


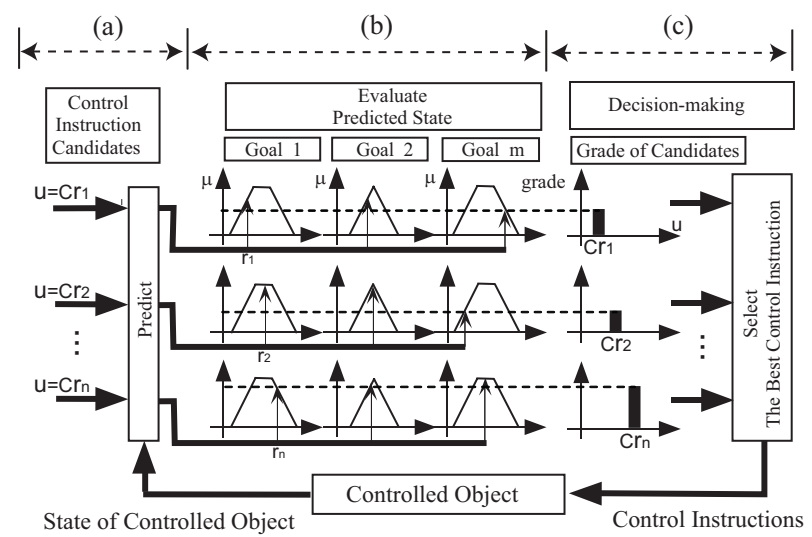

Fig. 5. Predictive fuzzy control

intelligent controller with a similar mechanism to predictive control based on expert's knowledge and experiences can be achieved with PFC.

Obviously, PFC has the three main characteristics of prediction, evaluation and decision in human's action decision. PFC method provides a potential means to realize human's prediction, evaluation and optimal decision-making process to select the best target element from a series of control instruction candidates by using FMCDM.

\subsubsection{Fuzzy Decision-Making by PFC}

MCDM is not only an efffective means for management decision but also valid for action decision for mobile body although there are not much applications until now. As described in the previous section, it should be noted that PFC can be regarded as a typical realization method of FMCDM with integrating predictive control, fuzzy logic and decision-making theories.

Let's consider the example in Fig. 4 (a) again, as a constraint can be regarded as a "goal" which satisfies the constraint, it is possible to convert all constraints to goals and expand them to $\mathrm{m}$ goals like Fig. 4 (b). Assume in a discrete decision space, let $R=\left\{r_{1}, r_{2}, \cdots, r_{n}\right\}$ be the set of decision alternatives, $\tilde{G}_{j}$ be the fuzzy sets representing control goal $j$. When the attainment of the goal $\tilde{G}_{j}$ by alternative $r_{i}$ can be expressed by the degree of membership $\mu_{\tilde{G}_{j}}\left(r_{i}\right)$, combining with the PFC architecture in Fig. 5, the decision set $\tilde{D}$ can be expressed as follow,

$$
\begin{array}{r}
\tilde{D}\left(r_{i}\right)=\tilde{G}_{1}\left(r_{i}\right) \cap \cdots \cap \tilde{G}_{j}\left(r_{i}\right) \cap \cdots \cap \tilde{G}_{m}\left(r_{i}\right) \\
(i=1,2, \cdots, n ; j=1,2, \cdots, m)
\end{array}
$$

and correspondingly its membership expression is

$$
\mu_{\tilde{D}}\left(r_{i}\right)=\mu_{\tilde{G}_{1}}\left(r_{i}\right) \wedge \cdots \wedge \mu_{\tilde{G}_{j}}\left(r_{i}\right) \wedge \cdots \wedge \mu_{\tilde{G}_{m}}\left(r_{i}\right)
$$

More generally, we may express $\mu_{\tilde{D}}$ as below,

$$
\mu_{\tilde{D}}\left(r_{i}\right)=\sum_{j=1}^{m} \alpha_{j} \cdot \mu_{\tilde{G}_{j}}\left(r_{i}\right) \quad \sum_{j=1}^{m} \alpha_{j}=1
$$




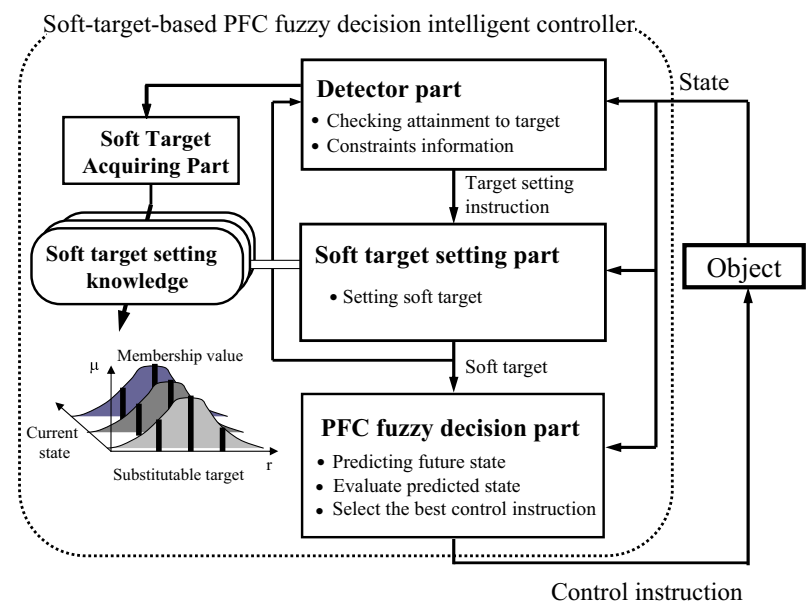

Fig. 6. Outline of soft-target-based fuzzy decision system

where weight coefficient $\alpha_{j}$ can be chosen in such a way to reflect the relative importance of each control goal $\tilde{G}_{j}$. It is consistent with fact since each control goal usually has different weight in a real system. Naturally, the $r_{i}$ with the highest membership degree will be selected as the optimal alternative. In short, a fuzzy decision-making may be completely realized by PFC.

\subsection{Soft Target Based PFC Fuzzy Decision}

The control instruction candidates of a conventional predictive fuzzy controller are usually acquired from experiences or try and error. They are limit and difficult to cover the whole control instructions domain. While if we get the control instruction candidates from the all current available target elements (acquired soft targets), the control instructions will more reasonable and have wider domain. This make the soft-target-based PFC fuzzy decision-making is greatly different from a conventional PFC.

The constructed PFC fuzzy decision-making system's configuration based on soft target is outlined as Fig. 6. It is composed of four parts: state detecting, soft target learning, soft target setting and fuzzy decision-making.

\subsubsection{Soft Target Learning Part}

For any sampling time, it is necessary to acquire all available sub-targets and their membership values based on the current state and environment information to prepare the soft target setting knowledge. It can be learned by final target based method or reinforcement learning method.

\subsubsection{Detector Part}

This part is used to detect the state variables and obstacles information, and judge the attainment degree to target (either sub-targets or final target) and the contact degree to obstacles. When it detected the previous sub-target was achieved or the vehicle can't move forward 


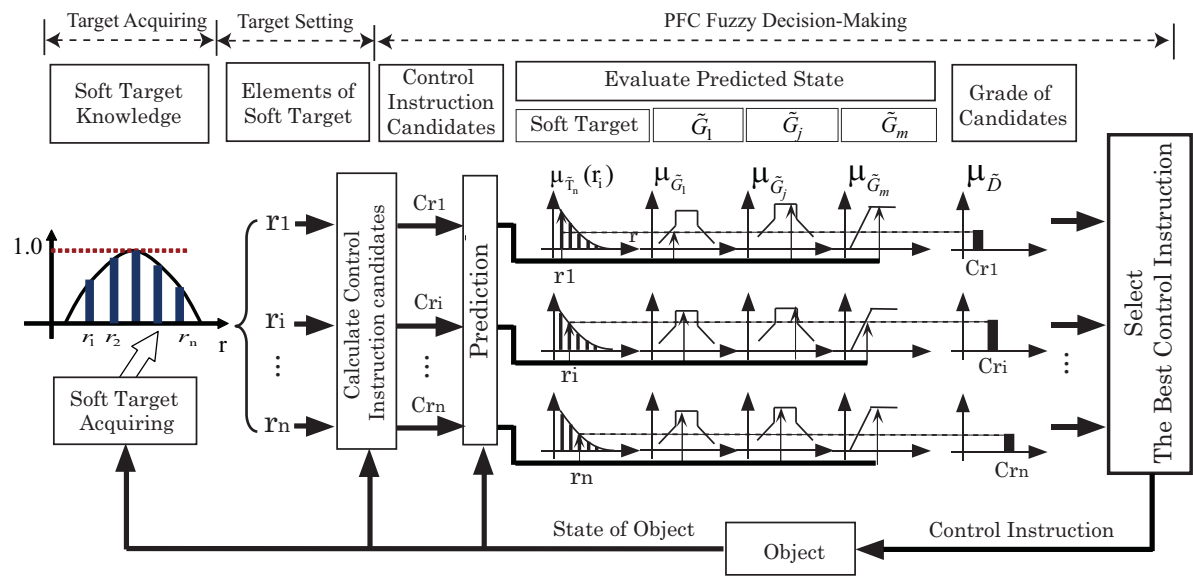

Fig. 7. PFC fuzzy decision-making with soft target

(or backward) anymore because of the influence of obstacles, target setting instruction is outputted to the soft target setting part.

\subsubsection{Soft Target Setting Part}

When target setting instruction is received, the soft target setting part carries out on-line learning for all possible candidates to calculate their membership values based object's current state and around obstacle information. It provides all possible candidates to fuzzy decision-making part to calculate control instruction candidates.

\subsubsection{Fuzzy Decision-Making Part}

Fuzzy decision is made by PFC as following process just like human's decision. It is easier to understand by combining Fig. 7. Firstly, each element of soft target is assumed as the control target, and the operation instruction candidate to each target is calculated. Next, the future state of controlled object is predicted by using all the operation instruction candidates in parallel. Then the future state is evaluated by fuzzy inference, and the evaluation value of the operation instruction candidate is calculated by equation (5) or (6). Lastly, the operation instruction candidate with the highest evaluation value is selected and given to the object as a control instruction.

These operations are repeated in the whole control process until achieving the final target. Thus, the fuzzy decision based on soft target is realized.

\subsection{Summary}

Soft target expand the conventional single target to a fuzzy target set by using fuzzy logic. PFC fuzzy decision-making provides a potential means to realize human's prediction, evaluation and optimal decision-making process to select the best target element from a series of control instruction candidates by using FMCDM. Soft-target-based PFC fuzzy decision-making control makes the control instructions more reasonable and have wider domain, which makes it greatly different from a conventional PFC. It is possible to solve the motion control problem 


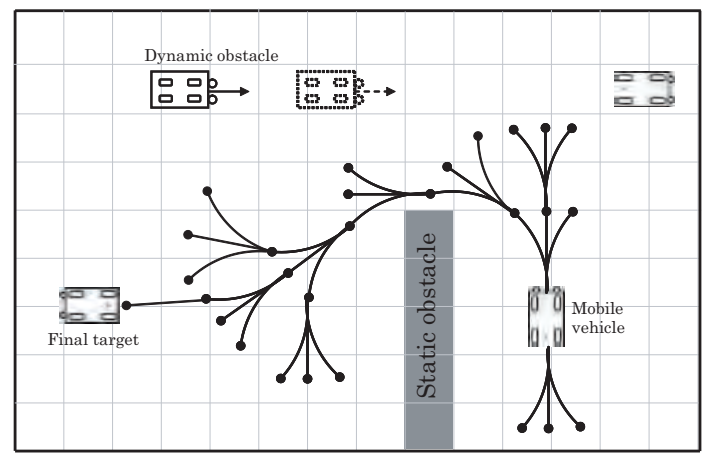

Fig. 8. Problem description

of mobile body with dynamic external constraints to achieve a flexible operation and selfadaptation to change situations.

\section{Application to Car-Like Mobile Vehicle}

The motion control problem of mobile vehicle can be typically formulated as planning a path between two specified locations, which is collision-free and satisfies certain optimization criterias. Autonomous moving in dynamic environment is still a very challenging problem because the surrounding situations are not qualified in static, knowledge is only partial and the execution is often associated with uncertainty. The car parking problem in a static environment has been studied by Prof. Yasunobu. An intelligent controller based on fuzzy target has been proposed. It solved the parking problem in a fixed space without moving obstacles. However, because the target is acquired off-line for a fixed parking lot, when the final goal or space changed, soft target had to been explored once more. It is difficult to respond to the dynamic environment such as moving obstacles, arbitrary placement of static obstacles or discretional initial position of the vehicle.

\subsection{Problem Description}

Four-wheeled car-like vehicle's motion control is very difficult because it has a strong internal constraint - minimal turn radius, which results in higher dependence of external environment and the demand of more well-rounded control knowledge. Let's consider a mobile vehicle moving in a narrow space in indoor environment with static and dynamic obstacles as denoted in Fig. 8 in which the dots means possible target candidates and the forking solid line means one moving route to one of the available multi-target candidates corresponding to the current state and obstacle information. The final target is able to be set as we want. The static obstacles can be placed at any position with arbitrary shape, and the vehicle can start at arbitrary initial position and orientation. In order to achieve a collision-free and low cost motion, the moving action from initial position to final target had to be designed online. Because of the nonholonomic characteristic and the impact of obstacles, it is necessary to find appropriate sub-targets corresponding to each current state and constraint information until arrive at the final target (Chen \& Yasunobu, 2008). 


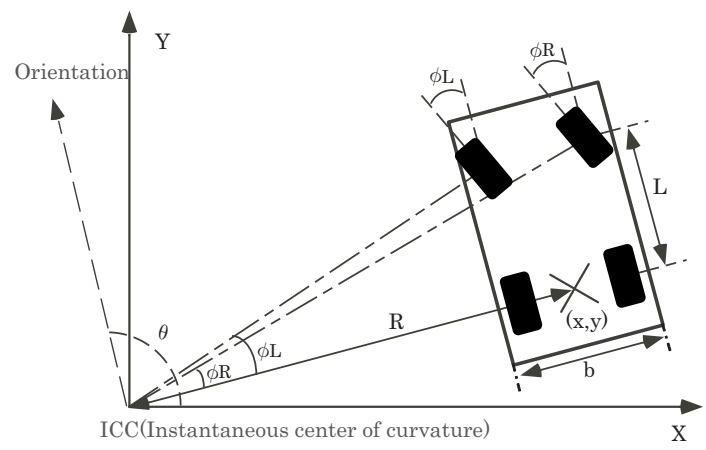

Fig. 9. Model of Ackerman steering mobile vehicle

\subsection{Four-Wheeled Mobile Vehicle Model}

Fig. 9 is the model of a car-like vehicle with Ackerman Steering. Where, $(x, y)$ is the Cartesian location of its rear wheels' center, $\theta$ is the heading angle between the body axis and the horizontal axis, $\phi_{L}$ and $\phi_{R}$ are relative steering angle of left and right wheel respectively, and $\phi=\left(\phi_{L}+\phi_{R}\right) / 2$ represents the steering angle with respect to the car body $\left(|\phi| \leq \phi_{\max }\right) . L$ is the wheelbase (longitudinal wheel separation). $b$ is the width of car (lateral wheel separation). $R$ is turning radius which is the distance from instantaneous center of curvature (ICC) to centerline of the vehicle. This system has 2 degrees of nonholonomy since the constraints on the system arise by allowing the wheels to roll and spin, but not slip. Thus, the Pfaffian constraints on the mobile vehicle become:

$$
\begin{array}{r}
\sin (\theta+\phi) \dot{x}-\cos (\theta+\phi) \dot{y}-L \cos \phi \cdot \dot{\theta}=0 \\
\sin \theta \cdot \dot{x}-\cos \theta \cdot \dot{y}=0
\end{array}
$$

Choosing $u_{1}=v \cos \phi$ and $u_{2}=\dot{\phi}$ as inputs yields:

$$
\dot{q}=\left(\begin{array}{c}
\dot{x} \\
\dot{y} \\
\dot{\theta} \\
\dot{\phi}
\end{array}\right)=\left(\begin{array}{c}
\cos \theta \\
\sin \theta \\
\frac{\tan \phi}{L} \\
0
\end{array}\right) u_{1}+\left(\begin{array}{l}
0 \\
0 \\
0 \\
1
\end{array}\right) u_{2}
$$

where, $v$ is the driving speed, $u_{1}$ corresponds to the translational velocity of the driving wheels and $u_{2}$ corresponds to the angular velocity of the steering wheels. Obviously, (8) is a socalled driftless nonlinear system with 2 inputs $(v, \phi)$ and 3 outputs $(x, y, \theta)$ constrained by $R_{\min }=L / \tan \phi_{\max }$. Where, $R_{\min }$ is the minimal turn radius, $\phi_{\max }$ is the maximal steering angle.

The parameters of the four-wheeled vehicle (assumed as about the same size of an RC car) are width of the car $b=18 \mathrm{~cm}$, wheelbase $L=25.6 \mathrm{~cm}$, minimal outside turning radius $R_{\min }=36.5 \mathrm{~cm}$, and the moving speed $v=10 \mathrm{~cm} / \mathrm{s}$ in both ahead and back. The map is set as Fig. 10, and the static obstacle is placed with different mode such as in the parking side, in opposite side and so on. The moving obstacle car with the same size of the controlled vehicle 


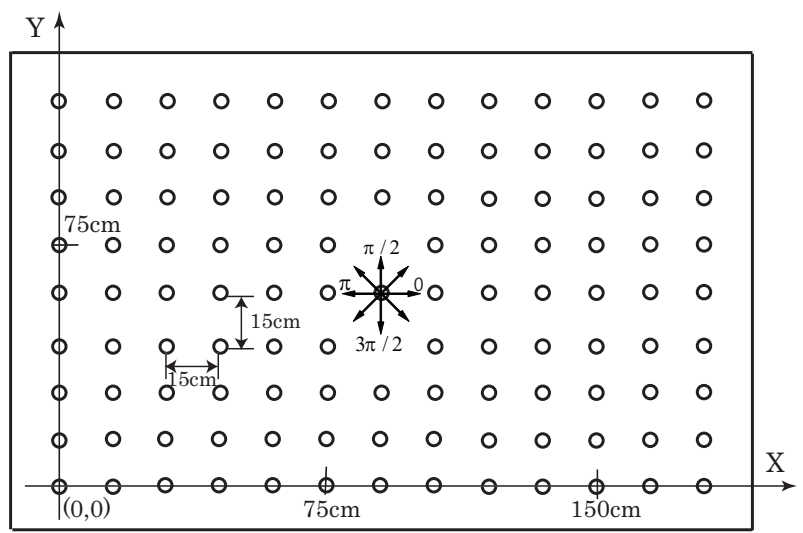

Fig. 10. Discretized moving space for four-wheeled vehicle

moves at a speed of $10 \mathrm{~cm} / \mathrm{s}$ from left to right with steering angle 0 . Final target and initial position can be set arbitrarily.

\subsection{Soft Target Acquisition}

In order to acquire the target setting knowledge for the current state, the $180 \mathrm{~cm} \times 120 \mathrm{~cm}$ space is described by occupancy grid maps with $15 \mathrm{~cm}$ interval as showed in Fig. 10 in which each small circle denotes a target location $(x, y)$ of vehicle (total $13 \times 9=117$ points). And the orientation $\theta$ is divided into eight azimuths $(0,0.25 \pi, 0.5 \pi, 0.75 \pi, \pi, 1.25 \pi, 1.5 \pi, 1.75 \pi)$. Thus, the space results $117 \times 8=936$ target candidates. For arbitrary state, we can approximate it to the nearest grid location and orientation. So it is possible to obtain all possible targets corresponding to the current state and obstacles information to form a state-action table with its action evaluation value named membership value in this study. This state-action table is defined as the soft target setting knowledge for the vehicle. It is acquired based on the final target, current state and obstacle information in real-time. The acquiring process is to find all possible sub-targets that can reach the final target directly (Chen \& Yasunobu, 2007a).

To obtain the evaluation value of each target, it is supposed that the vehicle moves from an arbitrary position $r_{i}=\left(x_{i}, y_{i}, \theta_{i}\right)$ in the space to achieve the final target $r_{\text {final }}=$ $\left(x_{\text {final }}, y_{\text {final }}, \theta_{\text {final }}\right)$ controlled by cascade fuzzy control method as showed in Figure 11 . In which, the current target orientation $\theta_{T}$ is fuzzy inferred from deflection $e_{X}$ of current position $X_{t}$ and target $X_{T}$, then, operation steering angle $\phi$ is fuzzy inferred from error $e_{\theta}$ of the target direction $\theta_{T}$ and the current body direction $\theta_{t}$. The membership functions used for evaluating $e_{X}$ and $e_{\theta}$ are denoted in Figure 12, where NB, NS, ZO, PS and PB are defined fuzzy linguistics variables corresponding with "Negative Big", "Negative Small", "Zero", "Positive Small" and "Positive Big" respectively. And the $1^{\text {st }}$ stage and $2^{\text {nd }}$ stage fuzzy inference models are denoted by Table 1 and Table 2 respectively.

The evaluation value $\mu_{\widetilde{T}_{n}}\left(r_{i}\right)$ is calculated according to the following cost functions. 


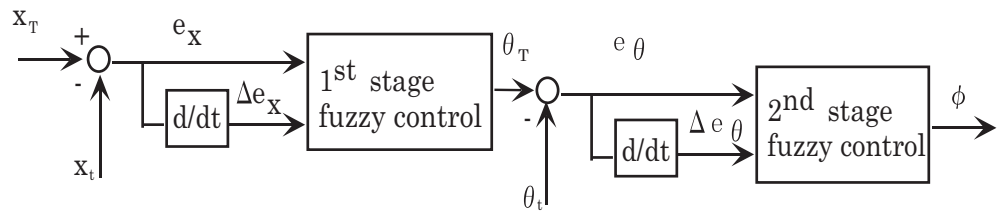

Fig. 11. Cascade fuzzy controller

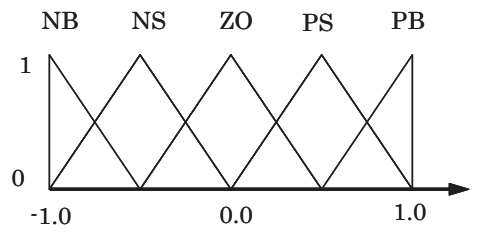

(a) $e_{x} / e_{\theta}$

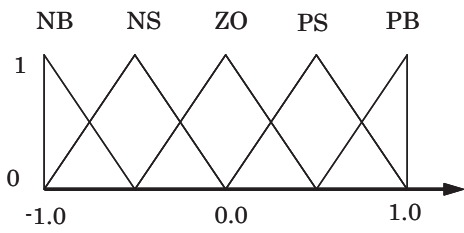

(b) $\Delta \mathrm{e}_{\mathrm{x}} / \Delta \mathrm{e}_{\theta}$

Fig. 12. Membership functions of cascade fuzzy controller

\begin{tabular}{c|c|c|c|c|c|c}
\hline \hline \multirow{2}{*}{$\theta_{T}($ rad $)$} & \multicolumn{5}{c}{$e_{X}(m)$} \\
\cline { 3 - 7 } \multicolumn{2}{c|}{} & NB & NS & ZO & PS & PB \\
\hline \multirow{4}{*}{$\Delta e_{X}$} & NB & -2.5916 & -2.4738 & -2.356 & -2.2382 & -2.1204 \\
\cline { 2 - 7 } & NS & -1.413 & -1.2958 & -1.178 & -1.0602 & -0.9424 \\
\cline { 2 - 7 } & ZO & -0.2356 & -0.1178 & 0.0 & 0.1178 & 0.2356 \\
\cline { 2 - 7 } & PS & 0.9424 & 1.0602 & 1.178 & 1.2958 & 1.4136 \\
\cline { 2 - 7 } & PB & 2.1204 & 2.2382 & 2.356 & 2.4738 & 2.5916 \\
\hline \hline
\end{tabular}

Table $1.1^{\text {st }}$ stage fuzzy inference model

\begin{tabular}{|c|c|c|c|c|c|c|}
\hline \multirow{2}{*}{\multicolumn{2}{|c|}{$\phi(\mathrm{rad})$}} & \multicolumn{5}{|c|}{$e_{\theta}(\mathrm{rad})$} \\
\hline & & NB & NS & $\mathrm{ZO}$ & PS & PB \\
\hline \multirow{5}{*}{$\Delta e_{\theta}$} & NB & 7.0 & 4.5 & 2.0 & -0.5 & -3.0 \\
\hline & NS & 6.0 & 3.5 & 1.0 & -1.5 & -4.0 \\
\hline & $\mathrm{ZO}$ & 5.0 & 2.5 & 0.0 & -2.5 & -5.0 \\
\hline & PS & 4.0 & 1.5 & -1.0 & -3.5 & -6.0 \\
\hline & PB & 3.0 & 0.5 & -2.0 & -4.5 & -7.0 \\
\hline
\end{tabular}

Table 2. $2^{\text {nd }}$ stage fuzzy inference model

$$
\begin{aligned}
\mu_{\widetilde{T_{n}}}\left(r_{i}\right) & =\mu_{\text {time }}\left(r_{i}\right) \wedge \mu_{\text {ope }}\left(r_{i}\right) \wedge \mu_{\text {err }}\left(r_{i}\right) \\
\mu_{\text {time }}\left(r_{i}\right) & =\left(t_{\text {max }}-t\right) / t_{\text {max }} \in[0,1] \\
\mu_{\text {ope }}\left(r_{i}\right) & =1.0-\alpha \sum_{t=0}^{\text {time }} \mid \text { ope }(t) \mid \in[0,1] \\
\mu_{\text {err }}\left(r_{i}\right) & =\mu_{d x}(x) \wedge \mu_{d y}(y) \wedge \mu_{d \theta}(\theta) \in[0,1]
\end{aligned}
$$




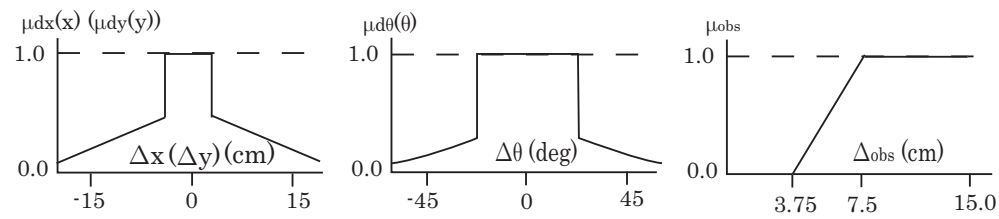

Fig. 13. Error evaluation membership functions

\begin{tabular}{c|l|c}
\hline \hline Number & Sub-target position & Membersip value $\mu$ \\
\hline 1 & $(15 \mathrm{~cm}, 15 \mathrm{~cm},-0.75 \pi)$ & 0.660 \\
\hline 2 & $(15 \mathrm{~cm}, 30 \mathrm{~cm},-0.75 \pi)$ & 0.596 \\
\hline 3 & $(15 \mathrm{~cm}, 45 \mathrm{~cm},-0.75 \pi)$ & 0.576 \\
\hline$\vdots$ & $\vdots$ & $\vdots$ \\
\hline 42 & $(60 \mathrm{~cm}, 105 \mathrm{~cm},-1.0 \pi)$ & 0.546 \\
\hline 43 & $(75 \mathrm{~cm}, 0 \mathrm{~cm}, 0.5 \pi)$ & 0.999 \\
\hline 44 & $(75 \mathrm{~cm}, 15 \mathrm{~cm}, 0.5 \pi)$ & 0.980 \\
\hline$\vdots$ & $\vdots$ & $\vdots$ \\
\hline 134 & $(165 \mathrm{~cm}, 90 \mathrm{~cm}, 0.25 \pi)$ & 0.497 \\
\hline 135 & $(165 \mathrm{~cm}, 90 \mathrm{~cm}, 0.5 \pi)$ & 0.464 \\
\hline 136 & $(165 \mathrm{~cm}, 105 \mathrm{~cm}, 0.25 \pi)$ & 0.475 \\
\hline \hline
\end{tabular}

Table 3. Soft target for final target $(75 \mathrm{~cm}, 0 \mathrm{~cm}, 0.5 \pi)$

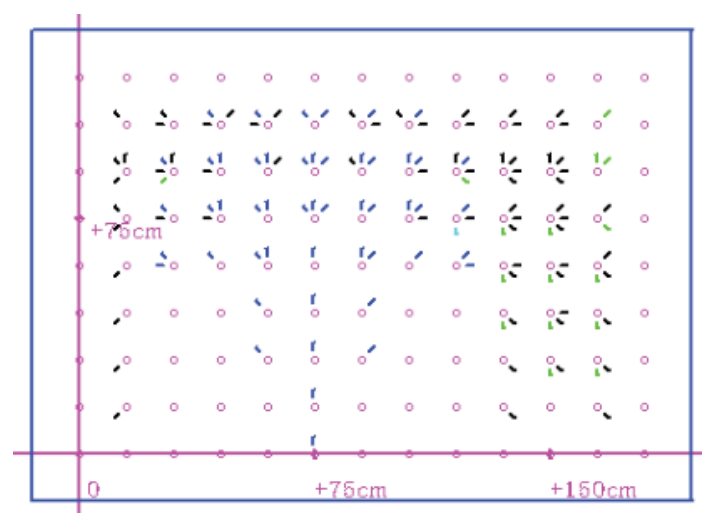

Fig. 14. Distribution map of soft target elements for final target $(75 \mathrm{~cm}, 0 \mathrm{~cm}, 0.5 \pi)$ with $\mu \geq 0.1$

Where, $\mu_{\text {time }}\left(r_{i}\right)$ is evaluation of moving time, $\mu_{\text {ope }}\left(r_{i}\right)$ is evaluation of steering amount, $\mu_{\text {err }}\left(r_{i}\right)$ is evaluation of arrival grade to final target. $t_{\text {max }}$ is the maximal limit time for a moving action, $t$ is the consumption time till arriving at the final target or being unable to move 


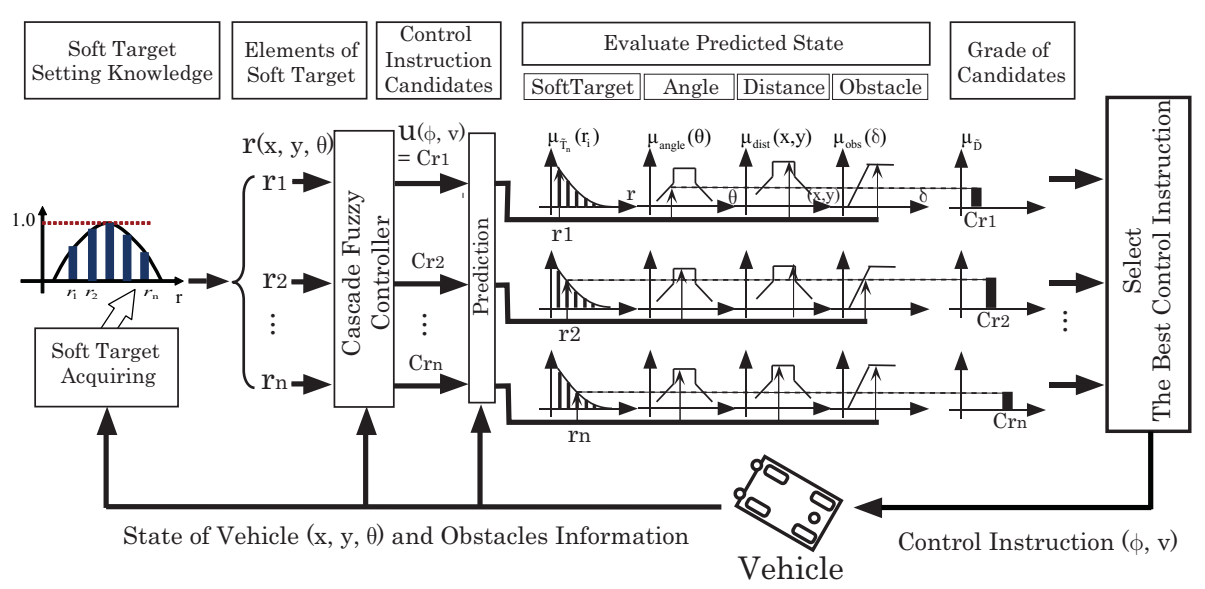

Fig. 15. PFC fuzzy decision with soft target for vehicle

anymore, $\sum_{t=0}^{\text {time }} \mid$ ope $(t) \mid$ is the total steering amount, $\alpha$ is coefficient of it. $\mu_{d x}(x), \mu_{d y}(y), \mu_{d \theta}(\theta)$ are error evaluations of current position $(x, y, \theta)$ to final target respectively whose error evaluation membership functions are shown in Figure 13. Here, $t_{\max }$ is set as $250 s$, and $\alpha$ is set as 1.5. They are estimated in experiences from the possible longest running time and maximal steering change amount. The less the consumption time or total steering amount or error evaluations to final target, the higher the evaluation value of the alternative target. For those that are unable to reach the final target, the membership values are set as 0 . For the first time, the soft target is acquired in this space without considering any obstacle, after then, it is acquired in an area near the vehicle current position (around $60 \mathrm{~cm}$ range) to reduce the computation expenses. If there is no available target for the current state, system selects the one acquired at the first time as the soft target set (Chen \& Yasunobu, 2009).

Based on the vehicle's kinematics model (8) and the cost evaluation function (9), we can obtain each available sub-target and its membership value which presents its satisfactory degree. Table 3 lists the acquired soft target set for final target $(75 \mathrm{~cm}, 0 \mathrm{~cm}, 0.5 \pi)$ without considering any obstacle from an arbitrary initial position in the 936 target candidates. There are 136 possible candidates in which $(75 \mathrm{~cm}, 0 \mathrm{~cm}, 0.5 \pi)$ has the highest evaluation value 0.999 . Figure 14 denotes the candidates who have the membership value no less than 0.1 . Here, blue cords denote the targets with membership value greater than 0.7 , black means the membership value is greater than 0.5 , green means greater than 0.3 , and magenta means greater than 0.1 .

\subsection{PFC Fuzzy Decision with Soft Target}

The constructed PFC fuzzy decision-making control system based on soft target for the fourwheeled car-like vehicle is denoted as Figure 15. Based on the vehicle's posture and environment information, state detector part decides whether giving target setting instruction or not by three flags. One is target achievement judgment flag which reflects whether the vehicle reached current target or not (judgment terms: $|\Delta x|<3.75 \mathrm{~cm},|\Delta y|<3.75 \mathrm{~cm}$, and $|\Delta \theta|<0.5 \mathrm{rad}$ ). The other two are moving flags which reflect whether the vehicle is able to move forward and backward respectively with judgment term $|\Delta o b s|<3.75 \mathrm{~cm}$ ). When it 


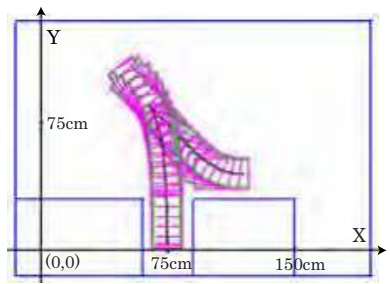

(a) Obstacles in parking side

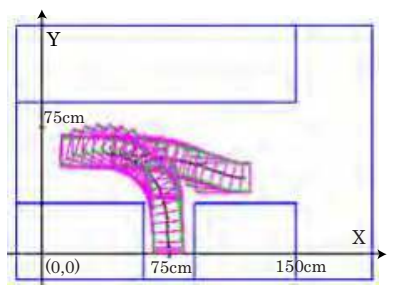

(b) Obstacles in parking and oppsite side

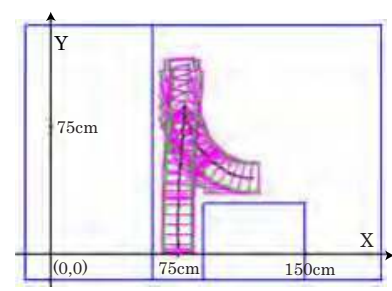

(c) Obstacles in parking and left side

Fig. 16. Parking simulation trajectories with different placement of obstacles

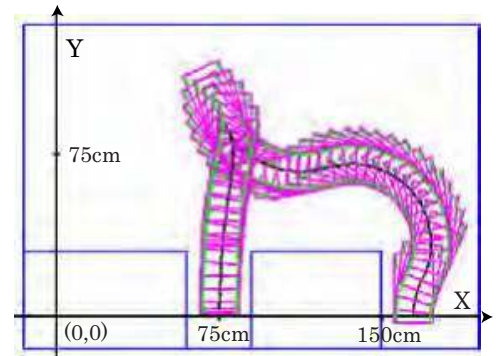

(a) Initial position $(165 \mathrm{~cm}, 0 \mathrm{~cm}, \pi / 2)$

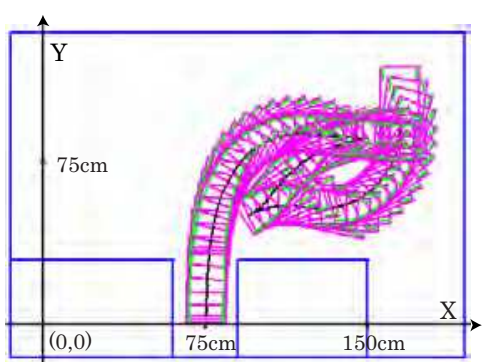

(b) Initial position $(165 \mathrm{~cm}, 90 \mathrm{~cm}, \pi / 2)$

Fig. 17. Simulation trajectories with different initial position

detected the previous sub-target was achieved or the vehicle can't move forward (or backward) anymore because of the influence of obstacles, target setting instruction is outputted to the soft target setting part. If it is necessary to reset target, soft target for the current state is obtained in target acquiring part to obtain all possible candidates.

For each candidate $r_{i}$ in $\widetilde{T_{n}}$, the control instruction $C_{r_{i}}$ is calculated by the cascade fuzzy control (Huang \& Yasunobu, 1999). And the future pose $\left(x_{t+1}, y_{t+1}, \theta_{t+1}\right)$ of vehicle is predicted for each instruction candidate $C_{r_{i}}$ by the kinematics model (8). Then multipurpose fuzzy evaluation is conducted for angle deflection, distance deflection and the minimal distance to obstacles. It calculates the evaluation values of all candidates and makes decision to select the one with the highest evaluation value as the control target by the following equation.

$$
\mu_{\tilde{D}}=\mu_{\tilde{T}_{n}}\left(r_{i}\right) \wedge \mu_{\text {angle }}(\theta) \wedge \mu_{\text {dist }}(x, y) \wedge \mu_{o b s}(\delta)
$$

The evaluation value of the operation instruction candidate which results moving in the opposite direction is reduced a half to make the vehicle select others' target candidates with higher evaluation to avoid trapping into dead loop of local minima.

\subsection{Simulation}

In order to confirm the validity of the constructed control system based on soft target, we carried out parking simulations with different placement of obstacles, arbitrary setting of initial position and final target, and autonomous running simulation with dynamic obstacle as well as static obstacles using the vehicle's parameters listed in 3.2. 


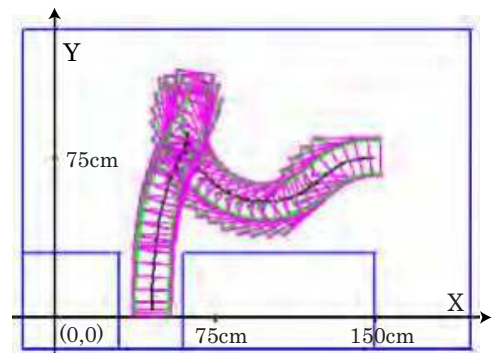

(a) Final target $(45 \mathrm{~cm}, 0 \mathrm{~cm}, \pi / 2)$

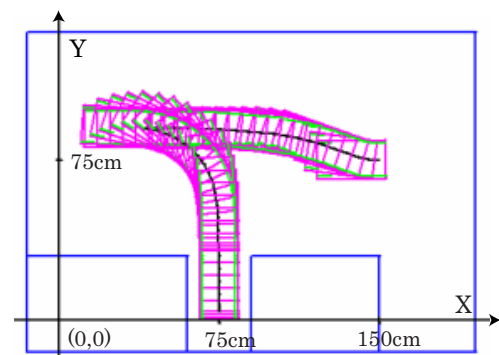

(b) Final target $(75 \mathrm{~cm}, 90 \mathrm{~cm}, \pi / 2)$

Fig. 18. Simulation trajectories with different final target

The coordinate of parking target point is set as $(75 \mathrm{~cm}, 0 \mathrm{~cm}, 0.5 \pi)$. Parking simulation is conducted in three kinds of suppositions of different obstacles placement. One is some parking lots in parking side are occupied, the other is parking side and opposite side are occupied, and another is parking side and its left side are occupied. The initial position for them is set as $(120 \mathrm{~cm}, 45 \mathrm{~cm}, \pi)$. Results are shown as Fig. 16. When parking lots in parking side are occupied, vehicle selects $(60 \mathrm{~cm}, 90 \mathrm{~cm}, 0.75 \pi)$ with the membership value $\mu=0.787$ as the best sub-target by acquisition and evaluation, and moves to final target as denoted in Fig. 16 (a). When parking lots in parking side and opposite side are occupied, vehicle selects $(30 \mathrm{~cm}, 60 \mathrm{~cm}, \pi)$ with $\mu=0.718$ as the best via-point, and achieves final target as shown in Fig. 16 (b). When parking lots in parking side and left side are occupied, vehicle selects $(90 \mathrm{~cm}, 60 \mathrm{~cm}, 0.5 \pi)(\mu=0.854)$ and $(75 \mathrm{~cm}, 90 \mathrm{~cm}, 0.5 \pi)(\mu=0.879)$ as sub-targets in turn, and moves to final target as Fig. 16 (c).

To confirm the control method's flexibility further, simulations with same final target but different initial position and same initial position but different final target were executed too.

Fig. 17 denotes the running trajectories for same final target $(75 \mathrm{~cm}, 0 \mathrm{~cm}, 0.5 \pi)$ from different initial position $(165 \mathrm{~cm}, 0 \mathrm{~cm}, 0.5 \pi)$ and $(165 \mathrm{~cm}, 90 \mathrm{~cm}, 0.5 \pi)$. For $(165 \mathrm{~cm}, 0 \mathrm{~cm}, 0.5 \pi)$, the vehicle achieved final target smoothly by selecting soft target element $(165 \mathrm{~cm}, 60 \mathrm{~cm}, 0.75 \pi)$ and $(60 \mathrm{~cm}, 90 \mathrm{~cm}, 0.75 \pi)$. But for $(165 \mathrm{~cm}, 90 \mathrm{~cm}, 0.5 \pi)$, the vehicle couldn't achieve final target by just once reverse. It firstly selected target $(90 \mathrm{~cm}, 60 \mathrm{~cm}, 0.25 \pi)$ to try to approach final target, but potential collision was predicted, so the vehicle reversed and select $(150 \mathrm{~cm}, 90 \mathrm{~cm}, 0 \pi)$ as sub-target to achieve a better via-points. Then it reversed again and reached final target successfully.

Fig. 18 shows the motion trajectories for different final target $(75 \mathrm{~cm}, 0 \mathrm{~cm}, 0.5 \pi)$ and $(45 \mathrm{~cm}, 0 \mathrm{~cm}, 0.5 \pi)$ from the same initial position $(150 \mathrm{~cm}, 75 \mathrm{~cm}, \pi)$. For final target $(75 \mathrm{~cm}, 0 \mathrm{~cm}, 0.5 \pi)$, the vehicle moved to left to approach the selected sub-target $(30 \mathrm{~cm}, 90 \mathrm{~cm}, \pi)$, and then reversed and arrived at final target quickly. But for $(45 \mathrm{~cm}, 0 \mathrm{~cm}, 0.5 \pi)$, because there is no enough space for the vehicle to reverse and park, membership value of $(30 \mathrm{~cm}, 90 \mathrm{~cm}, \pi)$ is decreased as it is close to the left wall. It is not the optimal one anymore. So the vehicle selected new optimum sub-target element $(30 \mathrm{~cm}, 90 \mathrm{~cm}, \pi)$ by fuzzy decision-making and achieved final target $(45 \mathrm{~cm}, 0 \mathrm{~cm}, 0.5 \pi)$ smoothly too.

From these results, it is confirmed that the vehicle controlled by this method can avoid the obstacles flexibly, and select the path with the lowest cost to achieve the task. And it can 


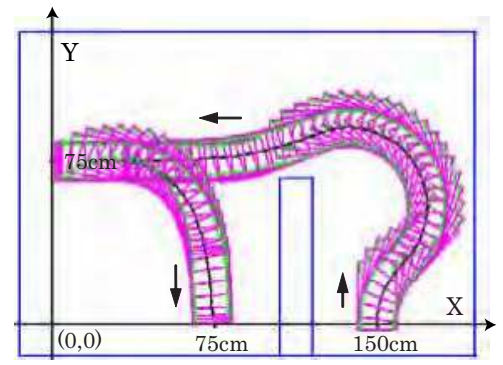

(a) With static obstacle

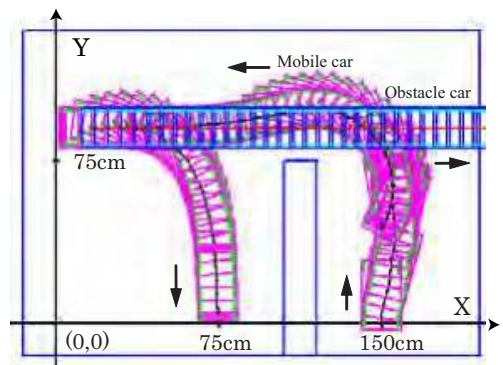

(b) With static and dynamic obstacles

Fig. 19. Simulation trajectories with static and dynamic obstacles

respond flexibly to different placement of obstacles, arbitrary setting of initial position and final target.

Though we mainly constructed parking experiments, it can be expanded to autonomous navigation easily because parking problem is in nature a highly autonomous navigation to achieve a special target or task.

A result example with static and dynamic obstacles is shown as Fig. 19. The initial position and final target are set as $(150 \mathrm{~cm}, 0 \mathrm{~cm}, 0.5 \pi)$ and $(75 \mathrm{~cm}, 0 \mathrm{~cm}, 0.5 \pi)$ respectively. The static obstacle is set as placed at $105 \mathrm{~cm} \leq x \leq 120 \mathrm{~cm}$ and $0 \mathrm{~cm} \leq y \leq 75 \mathrm{~cm}$. The dynamic obstacle is assumed as a mobile car with the same size of the controlled vehicle moving at a speed of $10 \mathrm{~cm} / \mathrm{s}$ from left to right with steering angle 0 . (a) is the moving trajectories with only static obstacle, the vehicle moves from initial position by choosing soft target elements $(165 \mathrm{~cm}, 30 \mathrm{~cm}, 0.25 \pi),(150 \mathrm{~cm}, 75 \mathrm{~cm}, 0.75 \pi),(30 \mathrm{~cm}, 60 \mathrm{~cm}, \pi),(30 \mathrm{~cm}, 75 \mathrm{~cm}, \pi)$, $(60 \mathrm{~cm}, 60 \mathrm{~cm}, 0.75 \pi)$, and $(75 \mathrm{~cm}, 0 \mathrm{~cm}, 0.5 \pi)$ in turn, and achieved final target smoothly. But when there are static and dynamic obstacles as (b), the vehicle tries to move like (a) firstly by selecting sub-targets $(165 \mathrm{~cm}, 30 \mathrm{~cm}, 0.25 \pi),(150 \mathrm{~cm}, 75 \mathrm{~cm}, 0.75 \pi)$, then it detected the moving obstacle car and had to reverse to guarantee safety by selecting optimun target element $(165 \mathrm{~cm}, 45 \mathrm{~cm}, 0.25 \pi)$. After it detected that the near range is safe (obstacle car have passed over), it moves to the final target again and achieved it successfully.

\subsection{Experiment}

In order to confirm the validity of proposed method further, we constructed the experiment system as denoted in Fig. 20. It is mainly composed of a control PC, a CCD camera, and a reconstructed vehicle from a RC car. The image of ceiling camera (KOCOM CCD camera) is transferred to PC by a USB capture board to calculate the vehicle's posture (position and orientation) and acquire obstacles' information with detection and tracking algorithm. Infrared sensors signals, tachogenerator signals and steering servo position are transmitted to PC by Bluetooth module. After integrating all acquired information, PC explores all available soft target elements for current state, and makes fuzzy decision to decide the next optimal viatarget, then calculate the steering and speed instructions, and then sends them to controlled vehicle by Bluetooth module. 


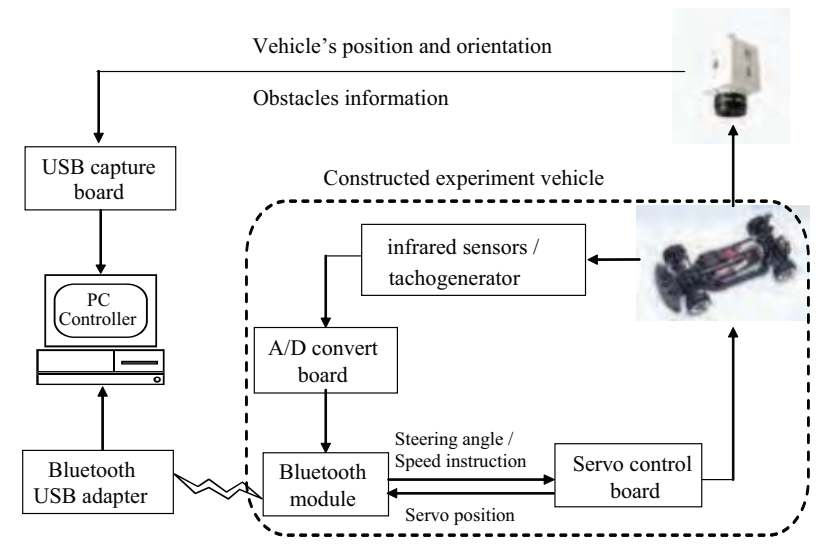

Fig. 20. Vehicle experiment system configuration

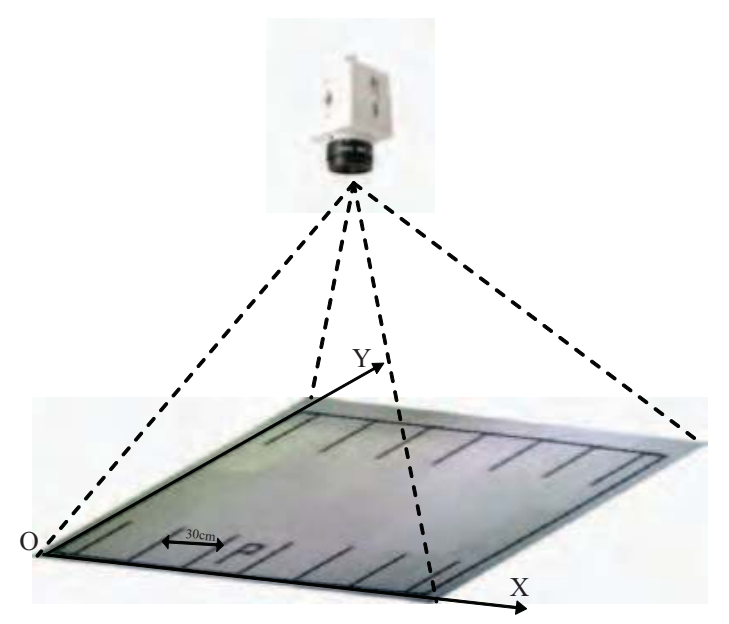

Fig. 21. Relationship of ceiling camera and world coordinate

\subsubsection{Camera Sensing}

In the experiment, a CCD camera hung on ceiling is used to capture the coordinate and orientation of controlled vehicle and all obstacles information in the space. As denoted in Fig. 21 , the motion space is on the ground of ICS laboratory. It is marked by some black adhesive tapes with the same size $180 \mathrm{~cm} \times 120 \mathrm{~cm}$ in simulation. Thus there are 12 units with the width $30 \mathrm{~cm}$. The unit with a letter "P" means where final target lies in. And like the simulation, a 15 $\mathrm{cm}$ margin distance is kept surrounding the edges of the space.

\section{Camera Calibration}

Camera calibration is a necessary step in 3D computer vision in order to extract metric information from $2 \mathrm{D}$ images. It is a process to determine the camera's parameters. Camera 


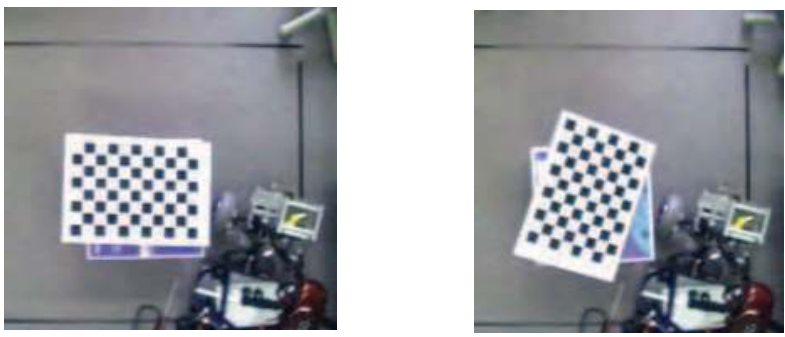

(a) Original sample images
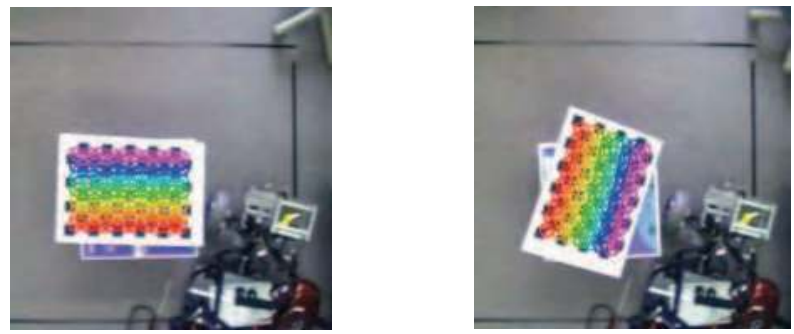

(b) Corners detection results

Fig. 22. Corners' detection results of some sample images

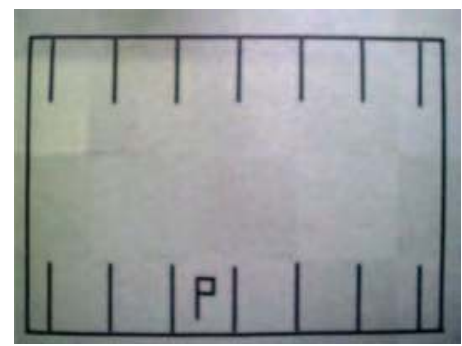

(a) Before calibration

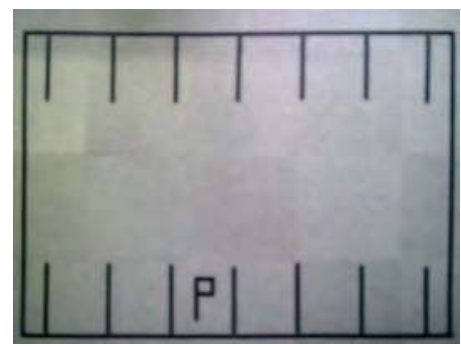

(b) After calibration

Fig. 23. Camera calibration

parameters are divided into intrinsic parameters and extrinsic parameters. Intrinsic parameters are used to specify the camera's characteristics. These parameters are: (1) focal length, i.e., the distance between the camera lens and the image plane; (2) the location of the image center in pixel coordinates; (3) the radial distortion coefficients of the lens. Extrinsic parameters are used to describe the relative spatial position and orientation between the camera and the world. These are the rotation matrix and translation vectors which determine the transformation between the camera and world reference frames (Wang \& Sugisaka, 2003).

Here photogrammetric calibration method is used, which is performed by observing a calibration object whose geometry in 3-D space is known with very good precision. 25 pictures with different placements of a $7 \times 10$ black and white chess board are used as calibration sample images. Some examples of chess board's corners detection image are shown in Fig. 22. The 


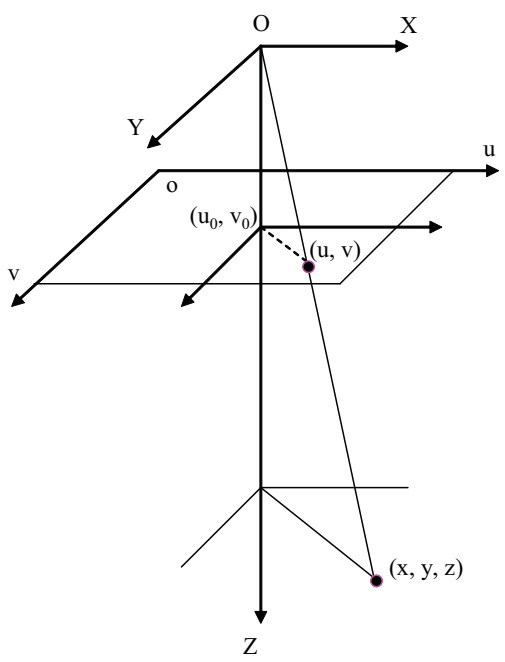

Fig. 24. Relationship of image coordinate and world coordinate

acquired intrinsic parameters matrix is

$$
\left[\begin{array}{ccc}
f_{x} & 0 & u_{0} \\
0 & f_{y} & v_{0} \\
0 & 0 & 1
\end{array}\right]=\left[\begin{array}{ccc}
427.96 & 0 & 123.75 \\
0 & 426.74 & 109.35 \\
0 & 0 & 1
\end{array}\right]
$$

Rotation matrix $R=[2.61,-0.72,-0.14]$. Translation matrix $T=[710.91,249.38,2.19 e+3]$. Lens distortion matrix is $[-0.23,0.32,-2.94 e-3,5.86 e-4]$. In the process of camera calibration, a distortion correction is also carried out. This is because straight lines in the world coordinate system are not straight in the image after perspective mapping. This would create errors when locating a point from the $2 \mathrm{D}$ image to $3 \mathrm{D}$ space. In the mapping process to correct any distortion, the distorted image taken directly with a CCD video camera is transformed into an undistorted image based on the radial distortion coefficient of the lens in lens distortion matrix. A comparison result of before calibration and after calibration with the acquired camera parameters are showed in Fig. 23. It is noted that the image distortions are greatly rectified after calibration, which guarantees the high precise of detection and tracking in following section.

Based on the acquired camera parameters, transform equation between world coordinate and image coordinate can be achieved from acquired translation rotation matrix, camera intrinsic matrix and lens distortion matrix. As shown in Fig. 24, the axis of the camera is thought of as being perpendicular, so the angle between the optical axes is not considered. Thus, the transform equation between world coordinate $(x, y, z)$ and image coordinate $(u, v)$ can be expressed as 


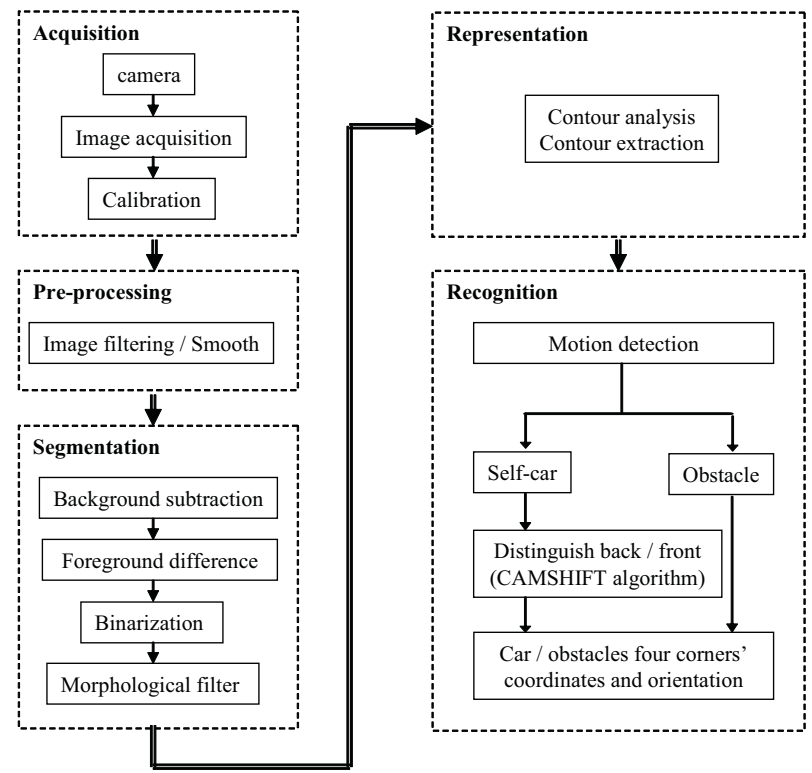

Fig. 25. Image processing flow chart

$$
s\left[\begin{array}{c}
u \\
v \\
1
\end{array}\right]=\left[\begin{array}{llll}
f_{x} & 0 & u_{0} & 0 \\
0 & f_{y} & v_{0} & 0 \\
0 & 0 & 1 & 0
\end{array}\right]\left[\begin{array}{cc}
R & T \\
0^{T} & 1
\end{array}\right]\left[\begin{array}{c}
x \\
y \\
z \\
1
\end{array}\right]
$$

where, $\mathrm{s}$ is a scalar factor. $\left(f_{x}, f_{y}\right)$ is focal length of the camera. $R$ is rotation orthogonal matrix. $T$ is translation matrix. $\left(u_{0}, v_{0}\right)$ is image center coordinate.

\section{Detection and Tracking}

To capture vehicle posture and all obstacles information, the camera is necessary to have the abilities to detect static or moving objects in the selected area, distinguish vehicle itself and static or dynamic obstacles, and distinguish back-front of the vehicle. The flowchart of detection and tracking algorithm is shown in Fig. 25. It is composed of five fundamental operations - acquisition, pre-processing, segmentation, representation, and recognition (Gini \& Marchi, 2002). Acquisition includes capturing the original image $(320 \times 240$ pixels $)$ of problem domain and calibrating it to rectify distortions and acquire the intrinsic and extrinsic parameters matrix. Pre-processing is used for image filtering and smooth processing. Segmentation covers background subtraction, foreground difference, binarization, and morphological filtering. Representation is for contour extraction - identifying objects shaped contours from edge data. Recognition includes objects detection (either static or dynamic), distinguishing vehicle itself and static or dynamic obstacles, and distinguishing back-front of the vehicle. Opencv ${ }^{1}$

\footnotetext{
${ }^{1}$ Refer to http:/ /opencv.jp/.
} 


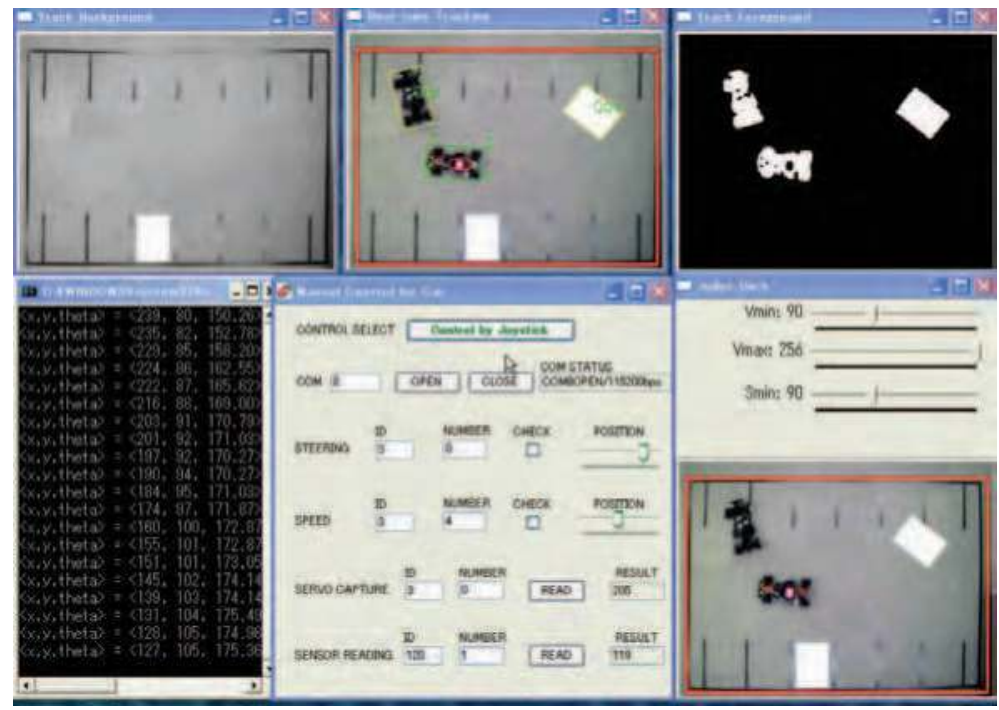

Fig. 26. Snapshot of detection and tracking

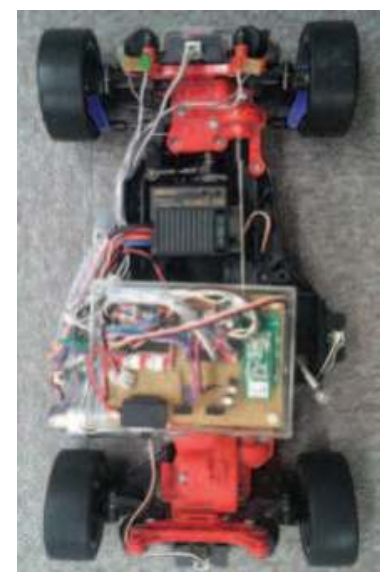

(a) Rebuilded vehicle internal structure

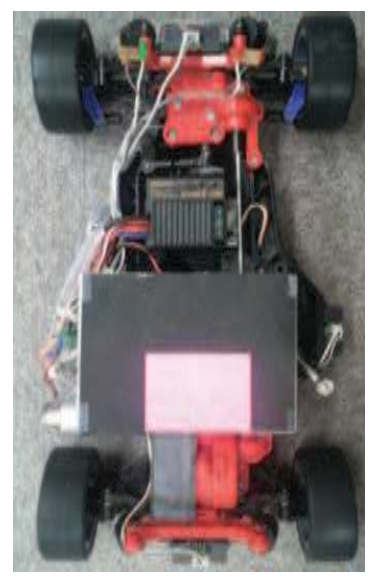

(b) Vehicle's back-front differentiation mark

Fig. 27. Rebuilded bluetooth vehicle for experiment

(a computer vision library originally developed by Intel.) is used as a base to construct the camera detection and tracking algorithm. And a precision with coordinate error less than $1 \mathrm{~cm}$ and orientation angle error less than $1^{\circ}$ was acquired. Fig. 26 displays a sample snapshot of detection and tracking result, which indicates both static and moving objects in the area are detected and tracked accurately. 


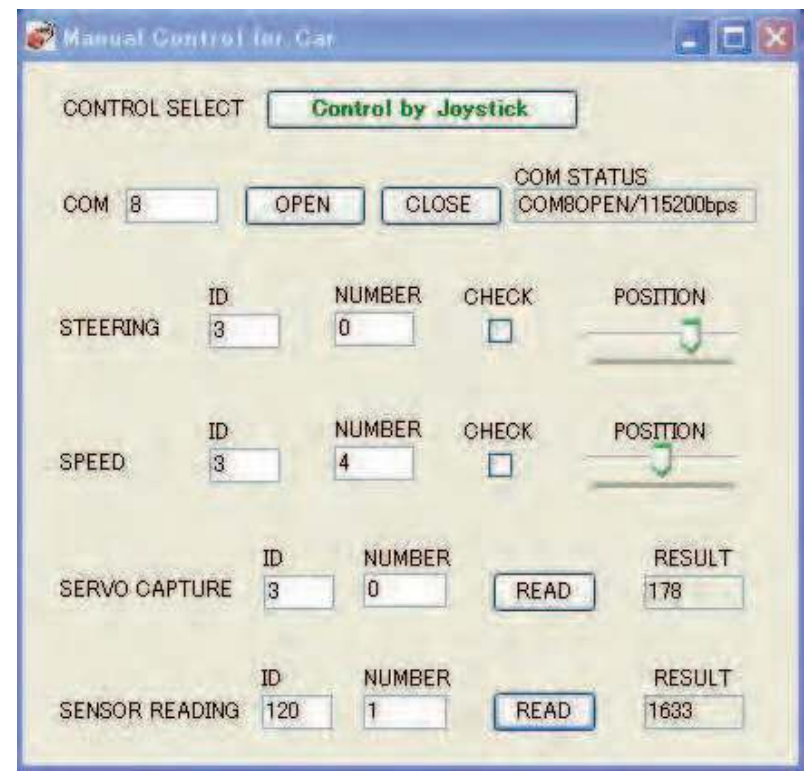

Fig. 28. Manual control interface

\subsubsection{Constructed Experiment Vehicle}

The experimental vehicle model (Fig. 27) is constructed by rebuilding a RC car (1/10 Calsonic Skyline GT-R Gr.A). It is mainly composed of a Bluetooth module, a servo control board and a A/D convert board. The original RC steering servo motor is replaced by KONDO's KRS$788 \mathrm{HV}$ servo motor with position capture function. The drive motor is replaced by a ESCAP motor-tachogenerator unit with a high gear ratio to guarantee the vehicle's speed unable to accelerate too high. Four infrared sensors (GP2D12) are equipped around vehicle's body to detect obstacles as a complementarity for camera sensing. A magenta mark is used to judge the detected object is the vehicle itself or obstacle, and distinguish back-front of the vehicle by CAMSHIFT (Continuously Adaptive Mean Shift) algorithm which is a simple color tracking algorithm widely used in motion tracking.

\subsubsection{Manual Control Interface}

To learn the vehicle control knowledge, including steering operation knowledge and speed control knowledge, a manual control interface (Fig. 28) is constructed. By this interface, the vehicle can be controlled by a joystick for steering, accelerating, braking, moving forward and backward, and the infrared sensors' value and steering servo motor position can be observed real-timely.

\subsubsection{Experiment Results}

As the same with simulations, we conducted experiments including same initial position and final target but different placement of obstacles, same initial position but different final target, and same final target but different initial position. A PC with the specifications $2.8 \mathrm{GHz}$ 


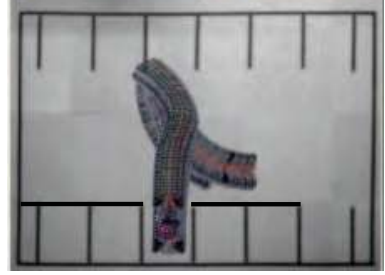

(a) Obstacles in parking side

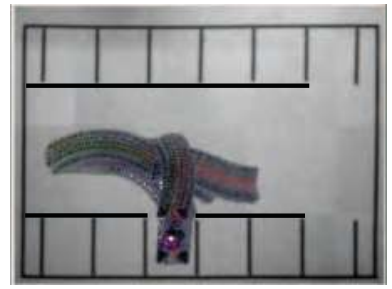

(b) Obstacles in parking and oppsite side

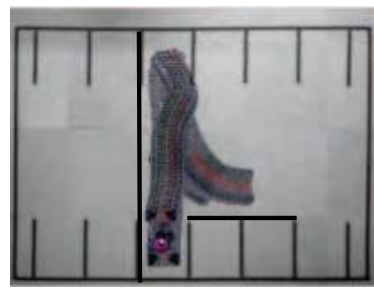

(c) Obstacles in parking and left side

Fig. 29. Parking experiment trajectories with different placement of obstacles

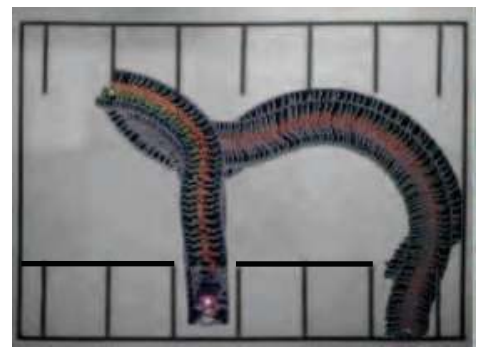

(a) Initial position $(165 \mathrm{~cm}, 0 \mathrm{~cm}, \pi / 2)$

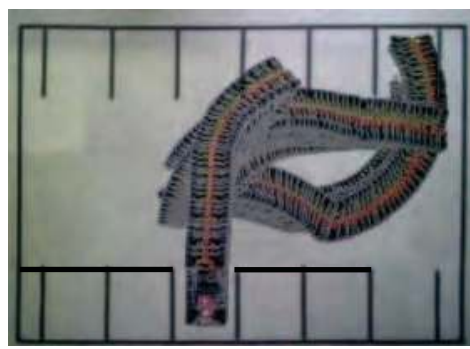

(b) Initial position $(165 \mathrm{~cm}, 90 \mathrm{~cm}, \pi / 2)$

Fig. 30. Experiment trajectories with different initial position

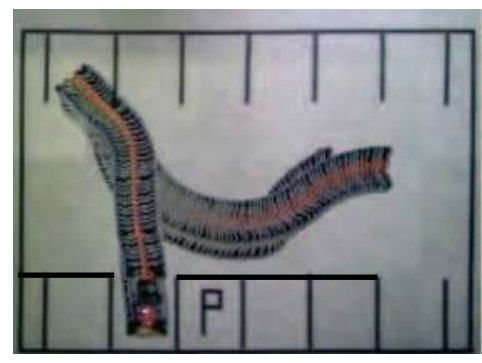

(a) Final target $(45 \mathrm{~cm}, 0 \mathrm{~cm}, \pi / 2)$

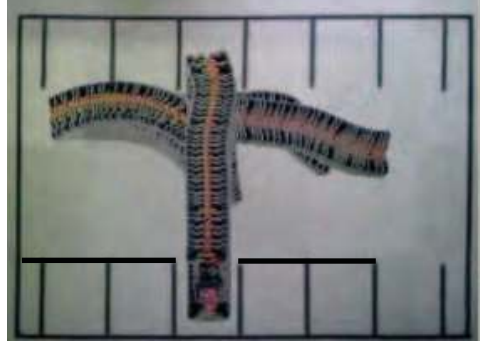

(b) Final target $(75 \mathrm{~cm}, 90 \mathrm{~cm}, \pi / 2)$

Fig. 31. Experiment trajectories with different final target

Celeron(R) CPU, $512 \mathrm{M}$ memory is used for simulation as well as experiment. The sampling time is set as $0.1 \mathrm{~s}$. The first time possible targets exploring for the whole space consumes about $100 \mathrm{~ms}$. And the possible targets exploring for the near domain of current position expends less than $20 \mathrm{~ms}$. Experiment test indicates the PC specifications is enough for current calculation amount including image processing.

The experiment results are shown in Figs. 29, 30, and 31. They are about the same with simulation results. For same initial position and final target but different placement of obstacles, 


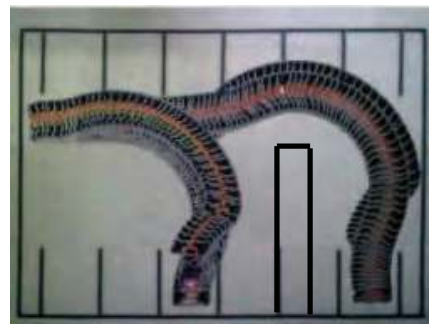

(a) With static obstacle

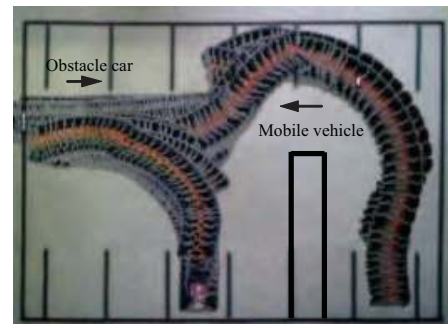

(b) With static and dynamic obstacles

Fig. 32. Experiment trajectories with static and dynamic obstacles

the elapsed times until finally reaching are $11.468 s, 17.515 s$, and $15.219 s$ respectively. The results indicate the controlled vehicle achieved task without restriction of obstacles' placement. For same initial position but different final target, the times of reaching the final target are $23.909 s$ and $33.502 s$ respectively. The controlled vehicle with flexible ability to reach any final target is demonstrated. For same final target but different initial position, the elapsed times until reaching final target are $18.314 s$ and $22.593 s$ respectively. It is clear that the vehicle can reached final target successfully from arbitrary initial position.

And from the results, we will find, in Fig. 29 (b) and Fig. 31 (b), the vehicle achieved the final target by selecting one more sub-target $(75 \mathrm{~cm}, 45 \mathrm{~cm}, 0.5 \pi)$ and $(75 \mathrm{~cm}, 90 \mathrm{~cm}, 0.5 \pi)$ than the simulations respectively. And in Fig. 30, the vehicle even had to choose two more subtargets to achieve the final target. It is considered that the camera sensing error and steering operation error which resulted in this tiny difference, but it didn't affect the achievement of the final target successfully. And on the other hand, it exactly demonstrated the flexibility of the proposed method more powerfully because of its ability to tolerate these errors and respond to uncertainties.

Fig. 32 denotes the running trajectories with static and dynamic obstacles. Here, the moving obstacle car is controlled by person with a remote controller. As it is powered by a line source rather than battery, the power line on the ground is easy to become a barrier for mobile vehicle. Thus the obstacle car is just controlled to move from left to center, and then reverse to return it start point. The mobile vehicle achieved the final target by few times reverses. The moving time until achieved final target is $27.249 s$ for with static obstacles and $49.686 s$ for with both static and moving obstacles. The results verify the vehicle can respond to dynamic environment flexibly.

These results verified the validity and flexibility of the proposed soft-target-based PFC fuzzy decision-making method for motion control of car-like mobile vehicle in changing situations more effectively.

\subsubsection{Discussions}

If there is no change in the environment, it is simple for vehicle making decision just to select the one with the highest membership value as control target until it reached the final target because the membership values will not change too. While in changing situation (with dynamic obstacle, change obstacle placement or add new static obstacle), the dynamic constraints are very possible to make it difficult to achieve the target (either sub-targets or final target) having the highest membership value because its evaluation value may decrease as the influence 
of constraints. It means the one with the highest membership value is unlikely the best one. Thus it is necessary to make decision to confirm which one is the best one in the alternative target set. We use PFC fuzzy decision-making method to simulate human's decision process to make dynamic fuzzy decision like human. Its advantages include:

- It is a multi-target-based rather than the conventional single-target-based method,

- Flexibility in responding to different optimization goals,

- Flexibility in responding to uncertainties,

- It simulated human's decision process completely and realized responding to changing situation flexibly,

- Vehicle is always supposed to choose the best one in the target set as control target, thus guaranteed the motion is collision-free and low cost,

- The soft-target-based PFC fuzzy decision-making control idea provides a new method for mobile body to realize dynamic environment or constraints self-adaptation.

\subsection{Summary}

Soft-target-based PFC fuzzy decision-making control for motion control of mobile vehicle (both two-wheeled differential drive vehicle and four-wheeled car-like Ackerman steering vehicle) with nonholonomic constraints in change situations is developed. From the simulation and experiment results (including same initial position and final target but different placement of obstacles, same initial position but different final target, same final target but different initial position, and with static and dynamic obstacles), it is learned that it is possible to achieve any final target and avoid obstacles (either static or dynamic) in the space flexibly without restriction of obstacle's placement and shape. A collision-free and low cost motion control of mobile vehicle with the ability of dynamic environment self-adaptation was achieved. A new method emulating the decision process of human for mobile body in dynamic environment was explored.

\section{Conclusions}

A soft-target-based fuzzy decision-making control for motion control of mobile vehicle with dynamic constraints was proposed. It emulates human's action decision process composed of prediction, evaluation and optimal decision-making based on multi-targets. The soft target defined as a fuzzy target set of all possible target candidates is learned based on final target, current state and constraints or environment information in real-time. For acquired soft target, fuzzy decision is made by predictive fuzzy control to select the best one as control target corresponding to the current state and constraints.

The proposed method was applied to motion control of a car-like mobile vehicle in a dynamic environment. The effectiveness and flexibility of this method was demonstrated by the simulation and experiment results. A collision-free and low cost motion control with the ability of dynamic environment self-adaptation was achieved. Parking in change situations (with different placement of static obstacles) and autonomous run with static and dynamic obstacle are realized. It is possible to achieve any final target from arbitrary initial position in a fixed narrow space without restriction of obstacle's placement and shape.

This soft-target-based fuzzy decision-making idea provides a new method for mobile body motion control in changing situation by simulating human's multi-target-based dynamic decision process. 


\section{References}

Barraquand, J., Langlois, B. \& Latombe, J. C. (1992). Numerical potential field techniques for robot path planning, IEEE Trans. Syst. Man Cybernet Vol. 22(No. 2): 224-241.

Bellman, R. E. \& Zadeh, L. A. (1970). Decision-making in fuzzy environment, Management Science Vol. 17(No. 4): B-141-164.

Chen, Y. G. \& Yasunobu, S. (2006). Soft target based intelligent controller for a system with dynamic restriction, Joint 3rd International Conference on Soft Computing and Intelligent Systems and 7th International Symposium on advanced Intelligent Systems, Tokyo, pp. 1949-1954.

Chen, Y. G. \& Yasunobu, S. (2007a). Soft target based obstacle avoidance for car-like mobile robot in dynamic environment, IEEE International Conference on Fuzzy Systems, London, pp. 1351-1356.

Chen, Y. G. \& Yasunobu, S. (2007b). Soft-target-based predictive fuzzy control for a cartpendulum system with dynamic constraints, Journal of Advanced Computational Intelligence and Intelligent Informatics Vol. 11(No. 8): 931-936.

Chen, Y. G. \& Yasunobu, S. (2008). Fuzzy target based soft decision for mobile vehicle in dynamic environment, 7th World Congress on Intelligent Control and Automation, Chongqing, pp. 800-805.

Chen, Y. G. \& Yasunobu, S. (2009). Soft decision with soft target for car-like mobile vehicle in dynamic environment, IEEJ Transactions on Electrical and Electronic Engineering Vol. 4(No. 4): $561-569$.

Gini, G. \& Marchi, A. (2002). Indoor robot navigation with single camera vision, Proc. of Pattern Recognition in Information Systems, PRIS, Alicante, Spain, pp. 67-76.

Huang, Y. \& Yasunobu, S. (1999). Cascade fuzzy control using to large scale system, Trans. IEE of Japan Vol. 119-C(No. 12): 1548-1553.

Jing, X. J. (2005). Behavior dynamics based motion planning of mobile robots in uncertain dynamic environments, Robotics and Autonomous Systems Vol. 53(No. 23): 99-123.

Kim, K. H. \& Cho, H. S. (2006). An obstacle avoidance method for mobile robots based on fuzzy decision-making, Robotica Vol. 24(No. 5): 567-578.

Ordonez, R. \& Zumberge, J. (1997). Adapitive fuzzy control: Experiment and comparative analyses, IEEE Transactions on Fuzzy Systems Vol. 5(No. 2): 167-188.

Wang, J. \& Sugisaka, M. (2003). Camera calibration for a mobile robot prototype, Artificial Life and Robotics Vol. 7: 91-94. 


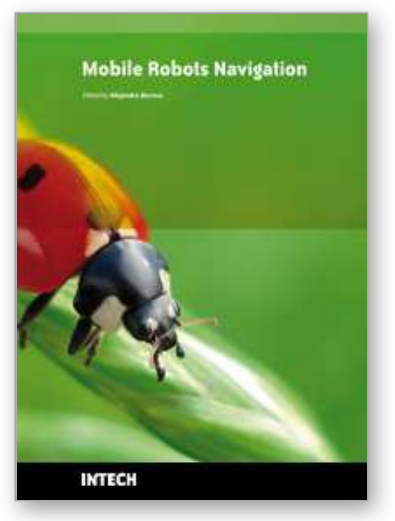

\author{
Mobile Robots Navigation \\ Edited by Alejandra Barrera
}

ISBN 978-953-307-076-6

Hard cover, 666 pages

Publisher InTech

Published online 01, March, 2010

Published in print edition March, 2010

Mobile robots navigation includes different interrelated activities: (i) perception, as obtaining and interpreting sensory information; (ii) exploration, as the strategy that guides the robot to select the next direction to go; (iii) mapping, involving the construction of a spatial representation by using the sensory information perceived; (iv) localization, as the strategy to estimate the robot position within the spatial map; (v) path planning, as the strategy to find a path towards a goal location being optimal or not; and (vi) path execution, where motor actions are determined and adapted to environmental changes. The book addresses those activities by integrating results from the research work of several authors all over the world. Research cases are documented in 32 chapters organized within 7 categories next described.

\title{
How to reference
}

In order to correctly reference this scholarly work, feel free to copy and paste the following:

You-gen Chen, Seiji Yasunobu, Wei-hua Gui, Ren-yongWei and Zhi-yong Li (2010). PFC Fuzzy DecisionMaking Control and Its Application to Car-Like Mobile Vehicle, Mobile Robots Navigation, Alejandra Barrera (Ed.), ISBN: 978-953-307-076-6, InTech, Available from: http://www.intechopen.com/books/mobile-robotsnavigation/PFC-Fuzzy-Decision-Making-Control-and-Its-Application-to-Car-Like-Mobile-Vehicle

\section{INTECH}

open science | open minds

\section{InTech Europe}

University Campus STeP Ri

Slavka Krautzeka 83/A

51000 Rijeka, Croatia

Phone: +385 (51) 770447

Fax: +385 (51) 686166

www.intechopen.com

\section{InTech China}

Unit 405, Office Block, Hotel Equatorial Shanghai

No.65, Yan An Road (West), Shanghai, 200040, China 中国上海市延安西路65号上海国际贵都大饭店办公楼 405 单元

Phone: +86-21-62489820

Fax: $+86-21-62489821$ 
(C) 2010 The Author(s). Licensee IntechOpen. This chapter is distributed under the terms of the Creative Commons Attribution-NonCommercialShareAlike-3.0 License, which permits use, distribution and reproduction for non-commercial purposes, provided the original is properly cited and derivative works building on this content are distributed under the same license. 